\title{
Le journal La Nature et la constitution de la collection de photographie scientifique du Conservatoire des arts et métiers
}

The journal La Nature and the constitution of the collection of scientific

photographs in the Conservatoire des arts et métiers

\section{Marie-Sophie Corcy}

\section{OpenEdition \\ Journals}

Édition électronique

URL : http://journals.openedition.org/dht/58

DOI : $10.4000 /$ dht.58

ISSN : $1775-4194$

Éditeur :

Centre d'histoire des techniques et de l'environnement du Cnam (CDHTE-Cnam), Société des élèves du CDHTE-Cnam

\section{Édition imprimée}

Date de publication : 1 décembre 2009

Pagination : 131-149

ISBN : 978-2-9530779-4-0

ISSN : 0417-8726

Référence électronique

Marie-Sophie Corcy, « Le journal La Nature et la constitution de la collection de photographie scientifique du Conservatoire des arts et métiers », Documents pour l'histoire des techniques [En ligne], $18 \mid 2^{\mathrm{e}}$ semestre 2009, mis en ligne le 06 avril 2011, consulté le 08 septembre 2020. URL : http:// journals.openedition.org/dht/58 ; DOI : https://doi.org/10.4000/dht.58 


\title{
Le journal La Nature et la constitution de la collection de photographie scientifique du Conservatoire des arts et métiers*
}

\author{
Marie-Sophie Corcy \\ Musée des arts et métiers - Cnam
}

\begin{abstract}
Résumé
Les acquisitions du Conservatoire des arts et métiers en matière de photographie scientifique sont représentatives de la politique que l'établissement a développée dans ce domaine à la fin du XIX` siècle. L'explosion des acquisitions photographiques a liev en 1881. Elle correspond à l'apparition du procédé au gélatino-bromure d'argent, qui va répondre aux besoins de la pratique de la photographie et permettre la naissance d'une industrie photographique, comme en témoigne l'explosion des dépôts français de brevets puis de marques de 1880 à 1914. Cette période va être prépondérante dans la constitution de la collection de photographies du Conservatoire. On dénombre l'acquisition de plus de deux mille objets ou images (soit une moyenne d'environ soixante-dix acquisitions par an). Quelles sont les raisons de ce développement et quels ont été les moyens mis en œuvre pour asseoir cette politique ? L'étude des conditions dans lesquelles s'est constitué le fonds de photographies scientifiques du musée confirme les liens que le Conservatoire des arts et métiers entretenait avec les milieux scientifiques et les sociétés savantes au XIXe siècle. Mais elle fait apparaître un axe inédit : le rôle du périodique de vulgarisation des sciences et techniques La Nature.
\end{abstract}

Résumés et mots clés en anglais sont regroupés en fin de volume, accompagnés des mots clés français

es premières acquisitions du Conservatoire des Larts et métiers en matière de photographie sont tardives. On pourrait s'attendre, compte tenu des liens existant entre les milieux techniques et scientifiques et le Conservatoire, à constater l'entrée de daguerréotypes - images ou matériel-dès 1839, année de la divulgation du procédé par François Arago (17861853) et Louis Jacques Mandé Daguerre (1787-1851). En réalité, les acquisitions de photographies sont très ponctuelles sur la période 1840-1880; on dénombre, en moyenne, seulement deux à trois acquisitions d'objets ou d'images par an.

L'explosion des acquisitions photographiques a lieu en 1881. Elle correspond à l'apparition du procédé au gélatino-bromure d'argent, qui va répondre aux besoins de la pratique de la photographie et permettre la naissance d'une industrie photographique, comme en témoigne l'explosion des dépôts français de brevets puis de marques de 1880 à 1914. Cette période va être prépondérante dans la constitution de la collection de photographies du Conservatoire. On dénombre
I'acquisition de plus de deux mille trois cents objets ou images (soit une moyenne d'environ soixante-dix acquisitions par an). Quelles sont les raisons de ce développement et quels ont été les moyens mis en œuvre pour asseoir cette politique?

\section{Contexte de la constitution des collections}

À l'image des collections de télégraphie qui s'enrichissent de façon cohérente au fur et à mesure des innovations techniques et des applications industrielles, on peut se demander sile développement des applications de la photographie, et notamment des applications scientifiques, n'a pas constitué une motivation encourageant les acquisitions dans ce domaine.

L'arrivée d'Aimé Laussedat (1819-1907) à la direction du Conservatoire en 1881 et la volonté d'organiser une " galerie de photographie ", dont l'initiative revient à son prédécesseur Hervé Mangon (1821-1888), expliquent également le développement des acquisitions. Le Conservatoire met en place une

* Cet article inaugure une nouvelle rubrique récurrente dans laquelle le Musée des arts et métiers présentera des études consacrées à l'histoire des techniques prenant appui sur ses collections patrimoniales ou sur celles conservées dans d'autres institutions en France et à l'étranger. 


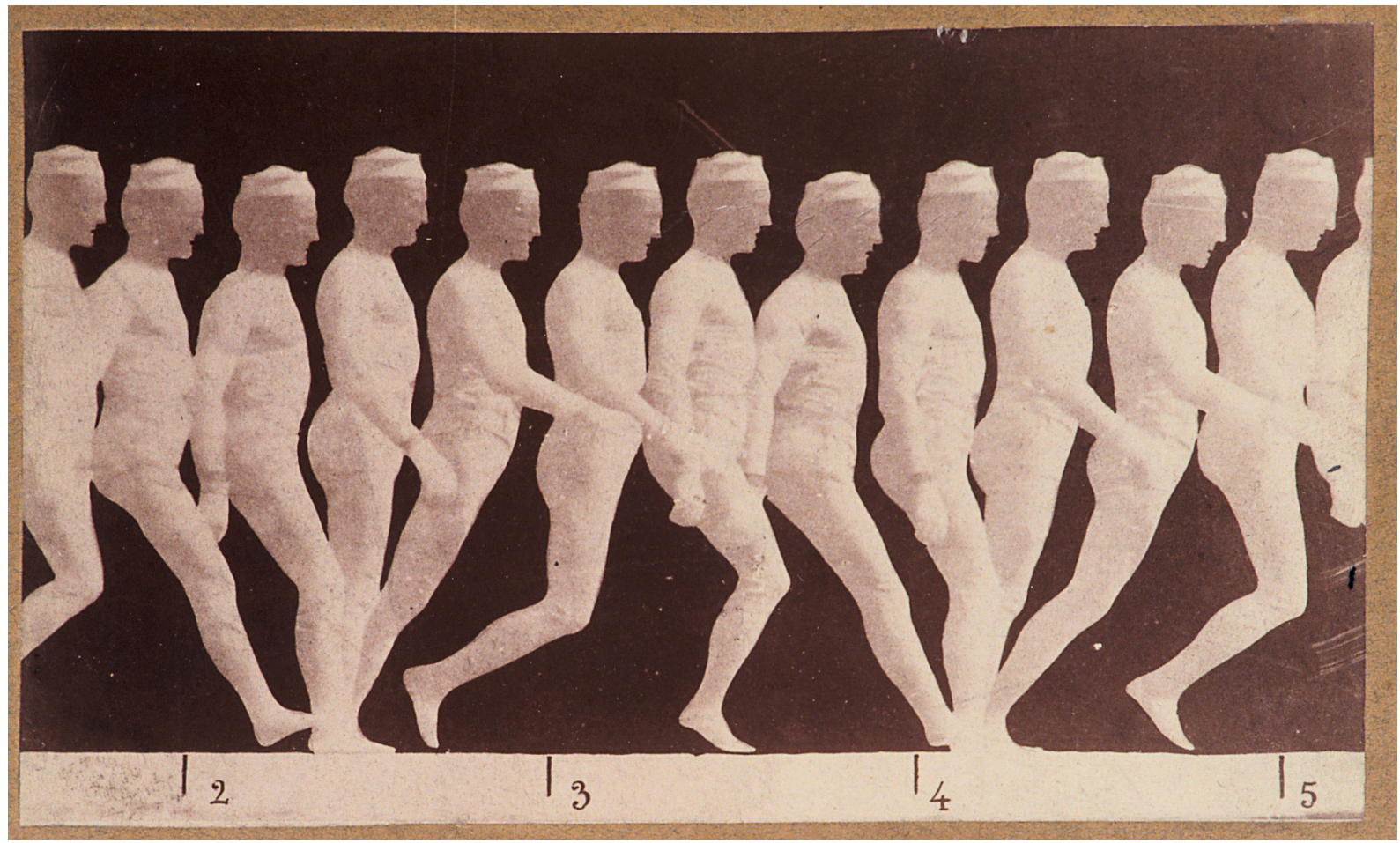

Vue chronophotographique: analyse de la locomotion humaine, Étienne Jules Marey, vers 1885. Épreuve positive sur papier albuminé. Inv. 9820-2-4. ๑ Musée des arts et métiers-Cnam / P. Faligot

politique d'acquisition volontariste comme l'indique cet extrait d'une lettre d'Aimé Laussedat à Aimé Civiale (1821-1893), le 18 mars 1882 :

"L'extension toute récente des galeries de collections du Conservatoire national des arts et métiers m'a permis de consacrer une série des salles aux appareils et aux œuvres susceptibles d'instruire les nombreux visiteurs de nos collections sur les divers procédés des arts graphiques. La photographie occupe naturellement une place des plus importantes dans cet ensemble'. ॥

Le chimiste Louis Alphonse Davanne (18241912)2 apparaît comme le conseiller scientifique de l'organisation de la galerie de photographie ; il sert également de lien entre le Conservatoire et les acteurs de cette histoire de la photographie que Mangon et Laussedat se proposent de mettre en scène. C'est

1 Musée des arts et métiers (après Mam), dossier d'œuvre (après DO) inv. 9696.

2 Lovis Alphonse Davanne se chargea de l'enseignement sur la photographie, dispensé dans le grand amphithéâtre de la Sorbonne en 1879, à l'initiative du conseil de I'Association scientifique de France-présidé par Milne Edwards-et de la Société française de photographie, dont il était à l'époque vice-président. dans ce contexte que se développent les collections de photographies scientifiques.

L'étude des correspondances contenues dans les dossiers d'œuvre fait apparaître la volonté manifeste d'accroître cet aspect des collections pour donner aux visiteurs " une idée exacte des progrès de la photographie ", comme l'atteste une lettre de remerciements adressée par Aimé Laussedat à Jules Janssen (1824-1907), pour le don d'épreuves:

« Je les joindrais aux premières et je ne doute pas que le public nombreux qui fréquente nos galeries serait très intéressé par les résultats que vous avez obtenus et qu'il n'en tire un grand profit pour son instruction ${ }^{3}$. ॥

\section{Les axes de développement}

Le fonds de photographies scientifiques concerne les disciplines suivantes: I'astronomie, la biologie, la médecine et l'histoire naturelle, la météorologie ou encore les recherches liées à la physique (comme la balistique ou l'étude des phénomènes électriques). Le protocole de réalisation des photographies emprunte aux techniques de la microphotographie, de la chronophotographie, de la stéréoscopie ou de la photographie de l'invisible.

Ce fonds se développe en cohérence avec le reste de la collection photographique (spécimens représentatifs des procédés et techniques

3 Mam, DO inv. 9764. Lettre du 16 juin 1882

132 Documents pour l'histoire des techniques $\mathrm{n}^{\circ} 18$ - décembre 2009 
photographiques; objets et machines photographiés) dont les pics d'acquisitions correspondent à la mise en place des présentations permanentes (dans les années 1880-1900 et 1926-1927).

La constitution des collections photographiques du Conservatoire s'est opérée selon deux axes que nous avons mis en évidence :

- un axe technique : l'image entre dans les collections car son exécution présente une innovation technique (protocole mis en œuvre pour son exécution, performance technique) ; l'image apparaît comme le résultat d'un processus scientifique expérimental ;

- un axe documentaire : l'image de l'objet est un avatar scientifique de l'objet réel.

La photographie scientifique revêt donc plusieurs aspects : performance technique, résultat tangible d'une expérience et donc source d'informations et de diffusion des connaissances. Si la photographie scientifique trouve sa justification par rapport à ces deux axes, elle dépasse ce postulat. Trace matérielle d'une expérience, elle doit être également appréhendée par rapport aux collections liées à l'instrumentation scientifique, à l'origine des collections du Conservatoire.

\section{Le rôle de La Nature}

Comment se sont opérés les choix en matière d'acquisition? Les rapports entre les autorités du Conservatoire national des arts et métiers et les milieux savants sont avérés. Les dépouillements des dépôts français de brevets et de marques mais aussi du Bulletin de la Société française de photographie font apparaître les tendances en matière d'évolution des techniques photographiques et mettent en exergue les préoccupations des praticiens. L'étude de la presse de vulgarisation scientifique et technique, à travers les numéros de La Nature, Revue des sciences et de leurs applications aux arts et à l'industrie publiés entre 1873 et 1905 (période pendant laquelle la majeure partie des fonds de photographie scientifique sont acquis), nous a permis de formuler plusieurs hypothèses quant au rôle du Conservatoire en matière de veille technologique. Relayant les innovations présentées à l'Académie des sciences ou à la Société d'encouragement pour l'industrie nationale, et publiées dans des périodiques spécialisés comme Photo-Gazette, La Nature a fait office de "filtre " et a permis aux autorités du Conservatoire d'opérer des sélections pour compléter les collections. Nous avons ainsi établi ce lien entre la publication, dans La Nature, d'articles concernant les applications scientifiques de la photographie et les acquisitions des épreuves dont il est question. Mais le lien est réversible, puisqu'au travers de ses articles,
La Nature a également conforté des choix d'acquisition du Conservatoire.

L'examen des modalités d'entrée des quelques objets $^{4}$ qui mentionnent le nom du fondateur de La Nature, Gaston Tissandier (1843-1899), nous renseigne déjà sur les liens que ce dernier entretenait avec Aimé Laussedat ${ }^{5}$. L'article d'Henri de Parville, à la suite du décès de Gaston Tissandier en 1899, nous indique que ce dernier entra au Conservatoire des arts et métiers dans le laboratoire du chimiste agronome Pierre Paul Dehérain (1830-1902). On y apprend également qu'il était « membre de la plupart des Sociétés scientifiques de Paris ॥ et que la Société d'encouragement pour l'industrie nationale lui décerna la grande médaille d'or en 1893 à la suite d'un « rapport très favorable de M. le colonel Laussedat $11^{6}$. Albert Tissandier (1839-1906) ${ }^{7}$ cite quant à lui Hervé Mangon comme étant l'une de ses relations privilégiées dans l'article qu'il lui consacre la même année8.

C'est ainsi que La Nature a relayé les aménagements du Conservatoire. Un article de Gaston Tissandier publié en $1883^{\circ}$ est consacré à la « nouvelle galerie des arts graphiques ». Tissandier en livre cette description :

« Dans le fond de cette galerie on trouve enfin deux pièces consacrées à la photographie et à ses applications aux arts et aux sciences. Ici les spécimens abondent, et on ne se lasserait pas d'admirer tout ce qui se trouve exposé. Voici d'abord l'histoire de la photographie, retracée à l'aide des épreuves obtenues par les Niepce, les Daguerre, les Talbot, les Bayard et les Poitevin ; voici de magnifiques spécimens de panoramas et de levés photographiques, de photographies célestes, lune, soleil, éclipses, comètes, etc. ; voici encore des cadres tout remplis de curieux tableaux, reproduisant les principaux évènements de l'histoire de Paris, embâcles de la Seine, inondations, éboulements,

4 Inv. 10031, 10090, 10393, 10701, 10705, 10775, 12044, TG439. 5 Une lettre de Gaston Tissandier à Aimé Laussedat, datée du 8 mars 1884 et conservée dans le dossier d'œuvre inv. 10090, donne le ton des relations qu'entretenaient les deux hommes (" Mon cher Colonel »). On y apprend par ailleurs que Tissandier sert d'intermédiaire quant à l'acquisition de l'épreuve de photographie aérienne réalisée par l'Anglais Cecil V. Shadbolt.

6 Henri de Parville, « Gaston Tissandier », La Nature, $2^{\mathrm{e}}$ semestre 1899, pp. 225-227.

7 Albert Tissandier était le frère de Gaston Tissandier.

8 Albert Tissandier, « Gaston Tissandier, sa vie intime », ibidem, pp. 248-250.

9 Gaston Tissandier, "Le Conservatoire des Arts et Métiers, la nouvelle galerie des arts graphiques "I, La Nature, $1^{\text {er }}$ semestre 1883, p. 267. 
souvenirs du siège, monuments célèbres, etc., objets naturels microscopiques amplifiés par la photographie, etc., etc. Ces galeries s'enrichissent par les dons qui sont adressés au Conservatoire, par des constructeurs ou des artistes, et la galerie des arts graphiques s'accroît et s'accroîtra sans cesse. »

\section{Les axes thématiques}

L'astronomie

"C'est à partir du moment où la photographie a reçu d'importantes applications à l'astronomie qu'elle a commencé à être comptée comme un art utile, respectable, pouvant être cultivé par des hommes de sciences. Jusqu'à ses applications astronomiques, la photographie était délaissée, contestée. Les portraits et les paysages, tel était le domaine où l'opinion publique la considérait comme à jamais confinée ${ }^{10}$. ॥ Si l'on se réfère à cette citation de Jules Janssen, il est logique que la majorité des acquisitions en matière de photographie scientifique concerne l'astronomie. Ce sont aussi les acquisitions les plus récurrentes.

La réalisation de ces images relève de la performance technique. Elles dépassent le statut de document, qui leur est conféré. Le daguerréotype du Soleil' ${ }^{1}$, réalisé par Léon Foucault (1819-1868) et Hippolyte Fizeau (1819-1896) en 1845, a été obtenu en utilisant un obturateur à guillotine. L'élaboration de cet obturateur - et son apparition en tant que dispositif autonome au moment où l'obturation est encore assurée par le bouchon d'objectif - marque une avance de près de trente-cinq années sur le système technique photographique. On peut donc voir que la science devient le moteur de l'évolution de ce système technique qui n'était pas en mesure de répondre favorablement aux ambitions de la sphère scientifique.

L'astronomie est également l'application scientifique de la photographie dont les articles sont les plus fréquents dans La Nature. Les thèmes qui reviennent régulièrement nous renseignent sur les travaux des astronomes, notamment sur la question de la formation du Soleil et des planètes, et sur l'élaboration de la carte du ciel (une douzaine d'articles sont consacrés à cette question entre 1875 et 1900). Le sujet de la constitution de collections dans le domaine de l'astronomie est abordé dans un article d'Ernest Mouchez (1821-1892) 12, directeur de

10 Jules Janssen, "La Photographie astronomique ", conférence du 20 décembre 1891, Annales du Conservatoire national des arts et métiers, 2e série, tome IV , 1892, pp. 249-262. 11 Inv. 17551. Nous ignorons à ce jour le contexte de cette acquisition (legs Léon Foucault ?).

12 Ernest Mouchez, « Création d'un musée astronomique à
l'Observatoire de Paris, en 1879. L'astronome signale l'approbation par le ministre de l'Instruction publique du projet présenté par l'Observatoire de Paris pour " la création, dans cet établissement, d'une collection d'objets devant former dans l'avenir un musée astronomique ") où figureront des collections « de portraits des astronomes et des savants, de médailles, de dessins, gravures, photographies, des anciens instruments d'astronomie ou de physique du globe ", mais également des « modèles réduits des instruments actuellement en usage dans les observatoires étrangers et des installations particulières à ces observatoires qu'il y aurait utilité de faire connaître ». Mouchez offre en 1882 au Conservatoire une collection d'épreuves photographiques reproduites par le procédé de photolithographie ${ }^{13}$. Elle représente les aménagements extérieurs entrepris par l'observatoire qui allaient recevoir des instruments d'observation ef les installations du nouveau musée astronomique.

La photographie astronomique demeura jusqu'au début du $X X^{\top}$ siècle une spécificité des milieux scientifiques. Les conditions qu'elle supposait interdisaient une pratique amateur. L'apparition du procédé au gélatino-bromure d'argent, les progrès de l'optique et l'essor d'appareils photographiques à main performants incitèrent les amateurs à s'y risquer ponctuellement, à l'occasion d'éclipses ou de phénomènes particuliers. Mais il n'en demeurait pas moins une pratique marginale et seule la prévision d'éclipses ou le passage d'une comète incitaient à une telle pratique.

Daguerre avait échové en 1839 lorsqu'il voulut reproduire la Lune par le daguerréotype, à cause du manque de sensibilité de l'iodure d'argent. Il constata néanmoins une action des rayons lunaires sur cette substance ${ }^{14}$, ce qui fit espérer à François Arago $^{15}$ la possibilité de réaliser la carte du ciel, un des défis les plus ambitieux des astronomes à la fin du XIXe siècle. Les limites du procédé photographique furent très exactement déterminées et ses possibilités s'élargirent parallèlement à l'évolution de ses techniques. La photographie astronomique emprunta à la stéréoscopie et à la chronophotographie. La stéréoscopie permettait d'apprécier les volumes et d'enrichir l'interprétation.

En 1877, l'astronome Jules Janssen obtenait à l'Observatoire de Meudon des photographies de

l'Observatoire de Paris I), La Nature, 1er semestre 1879, pp. 262-263. 13 Inv. 9700.

14 François Arago, OEuvres complètes, tome VII, pp. 459-498. 15 François Arago, discours fait devant la Chambre des députés lors de la séance du 3 juillet 1839.

134 Documents pour l'histoire des techniques nº 18 - décembre 2009 


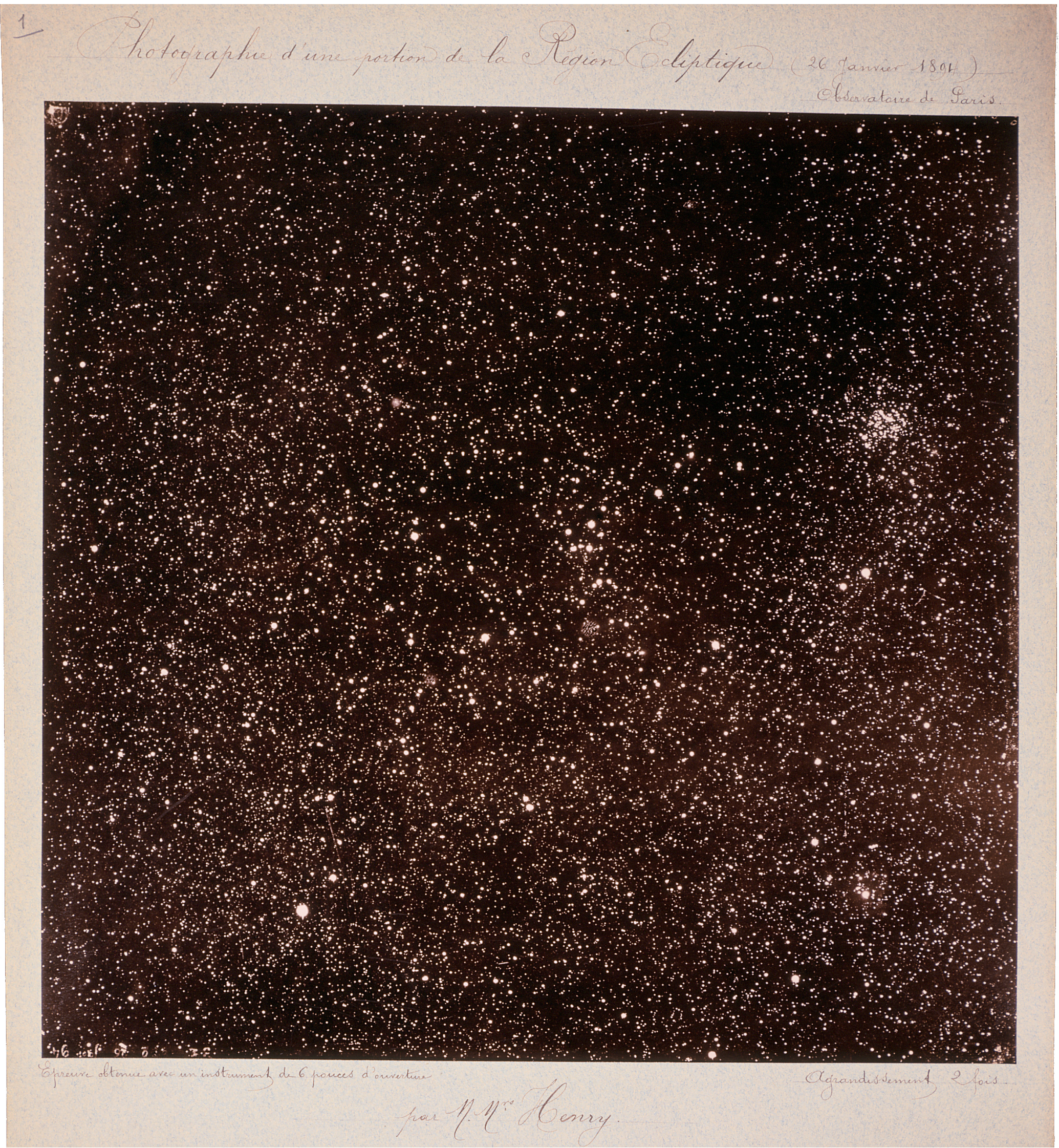

Carte photographique du ciel, vue d'une portion de la région écliptique obtenue le 26 janvier 1894 à l'Observatoire de Paris, Prosper et Paul Henry. Épreuve positive sur papier albuminé. Inv. TG3888-1 @ Musée des arts et métiers:Cnam /L. Karteskind 
la surface solaire ${ }^{16}$. Ces clichés se révélèrent utiles à la connaissance de la constitution physique de la photosphère. L'année suivante, Janssen rédigeait une note " sur des faits nouveaux touchant la constitution du soleil révélés par la photographie $11^{17}$. Janssen ${ }^{18}$ publie peu après un article sur la constitution de la surface solaire. Ses travaux seront encore mentionnés en 1886 19 . La série inv. 9534 concerne les taches et granulations solaires, mais elle comporte également des vues de comètes et de la Lune. Elle est offerte en 1881 par Janssen en réponse à la demande de Laussedat. La copie d'une lettre de ce dernier à l'astronome le 19 juin 1882 précise les circonstances du don :

" Je pense que ces indications vous suffiront pour vous guider dans le choix des autres épreuves qui seront de nature à donner aux visiteurs du Conservatoire une idée exacte des remarquables progrès de la photographie céleste. ॥

Précisons que Laussedat fait au préalable l'état des photographies célestes déjà données à l'établissement. Les travaux de Janssen ${ }^{20}$ relatifs à la photométrie sont illustrés dans la collection du musée par la présence du «Photomètre photographique " qu'il avait élaboré dans le cadre de ses recherches ${ }^{21}$.

Les collections du musée s'enrichissent en 1898 d'une série de clichés de la surface solaire ${ }^{22}$ exécutés par Maurice Lœwy (1833-1907)23 à l'Observatoire de

16 Louis Figuier, «L'Observatoire de Meudon etles découvertes d'astronomie physique dues à la photographie ", L'Année scientifique et industrielle, 1878, pp. 32-36.

17 Jules Janssen, Bulletin de la Société française de photographie, séance du 4janvier 1878, pp. 22-25. Voirégalement: Jules Janssen, "Sur la constitution de la surface solaire et sur la photographie envisagée comme moyen de découverte en astronomie physique ", Comptes rendus des séances de l'Académie des sciences, tome LXXXV, p. 1249 ; « Sur la constitution de la surface solaire et sur la photographie envisagée comme moyen de découverte en astronomie physique "), La Nature, 1er semestre 1878, pp. 154-155 et pp. 177-178; «Sur la photographie envisagée comme instrument de découverte en astronomie ", Comptes rendus des séances de l'Académie des sciences, tome Cll, p. 80 ; « La Constitution des taches solaires et la photographie en astronomie ॥, La Nature, $1^{\text {er }}$ semestre 1886, p. 111 , pp. 235-236.

18 Jules Janssen, "Sur la constitution... », art. cit.

19 Jules Janssen, "La constitution des taches solaires... », art. cit. 20 Jules Janssen, «Sur la photométrie photographique et son application à l'étude des pouvoirs rayonnants comparés du Soleil et des étoiles I), La Nature, 1 ${ }^{\text {er }}$ semestre 1881, p. 395-396. 21 Inv. 12703. 22 Inv. TG390.

23 Maurice Lœwy est directeur de l'Observatoire de Paris de
Meudon en 1877, 1885, 1887, 1890 et 1893. Auguste Fraissinet ${ }^{24}$ mentionnait les récents travaux de l'astronome dans un article publié en 1895.

La photographie de la Lune donne lieu à une série d'articles, régulièrement publiés de 1890 à 1898. L'épreuve de Lewis M. Rutherfurd (1816-1892) ${ }^{25}$ exécutée en 1865, entre dans les collections du Conservatoire à la suite de l'Exposition universelle de 1867. La nécrologie de l'astronome ne manque pas d'y faire référence :

« En 1865, il décrit son appareil photographique imaginé en vue des rayons chimiques. II indique son mode de construction et donne comme exemple des résultats obtenus sa magnifique photographie de la lune, remarquable par la finesse de ses détails ${ }^{26} . »$

De même, Fraissinet ${ }^{27}$, de l'Observatoire de Paris, mentionne en 1890 les travaux de Warren de La Rue (1815-1889) ${ }^{28}$, dont le Conservatoire a acquis des épreuves en 1869 ; mais au-delà de la communauté scientifique, ce sont bien les épreuves de Rutherfurd qui ont attiré l'attention du public :

"Mais ce sont celles de Rutherfurd, de New York, qui ont rendu le plus de services parce qu'elles ont été multipliées et se trouvent dans le commerce ; elles sont au nombre de trois de 53 centimètres de diamètre : deux des quadratures et une de la pleine lune. »)

Dans le même article, Fraissinet cite les « nouvelles photographies lunaires de MM. Henry "):

"La supériorité de ces résultats tient non seulement à la perfection de l'objectif photographique construit par MM. Henry et si habilement utilisé par eux, mais encore au procédé d'agrandissement direct qu'ils réalisent en adaptant un appareil spécial au foyer de la lunette. Les clichés obtenus

1897 à 1907.

24 Auguste Fraissinet, « Photographies lunaires récemment obtenues à l'Observatoire de Paris ॥, La Nature, $1^{\text {er }}$ semestre 1895, pp. 295-298.

25 Inv. 7880.

26 « Nécrologie - L.-M. Rutherfurd ", La Nature, $2^{\mathrm{e}}$ semestre 1892, pp. 174-175.

27 Auguste Fraissinet, "Photographies lunaires », La Nature, $2^{\text {e }}$ semestre 1890, pp. 104-106.

28 En 1858, Warren de La Rue faisait la démonstration de la sphéricité de la Lune au moyen du stéréoscope employé pour l'examen des clichés. Inv. 8040 et 8041.

136 Documents pour l'histoire des techniques $\mathrm{n}^{\circ} 18$ - décembre 2009 
dans ces conditions nouvelles donnent aux images une netteté beaucoup plus considérable que celle que donne l'agrandissement après coup d'un cliché ou d'une épreuve ${ }^{29} .11$

Les vues de la Lune de Prosper (1849-1903) et Paul (1848-1905) Henry ${ }^{30}$, exécutées vers 1890-1893, sont acquises par donation des astronomes en 1898. Fraissinet fait une nouvelle fois référence à leurs travaux en $1893^{31}$ et en 189532. Les clichés de Maurice Lœwy et Pierre Henri Puiseux (1855-1928) ${ }^{33}$ constituant l'Atlas de la lune, entrés dans les collections en 1898, sont cités par Fraissinet en $1895^{34}$; notons que les clichés de Warren de La Rue et de Lewis M. Rutherfurd servent toujours de référence à cette date :

"Avec ces dernières photographies [épreuves obtenues par Prosper et Paul Henry] et celles de MM. Lœwy et Puiseux, nous arrivons à des agrandissements photographiques dont l'échelle est équivalente à celle des plus grandes cartes de la lune; tandis qu'auparavant les photographies lunaires les plus connues, celles de Warren de La Rue et de Rutherfurd, n'avaient donné, à l'amplification, que des épreuves présentant une infériorité considérable par rapport à la vision directe. A l'aide des épreuves nouvelles, au contraire, on a pu rectifier les dessins les plus détaillés, apercevoir de nombreux petits cratères omis jusqu'à ce jour sur les cartes des plus habiles sélénographes; et, dans bien des cas, arriver à définir avec certitude l'aspect et l'existence d'objets contestés à la surface de notre satellite. [...] Disons en terminant qu'un grand nombre de clichés ont été déjà obtenus et que les agrandissements réalisés pourront être utilisés en vue de la formation d'un atlas lunaire qui mettra à disposition des astronomes et des géologues des documents précieux pour arriver à la solution des problèmes que soulève l'étude de la constitution de notre satellite. ")

29 Auguste Fraissinet, art. cit.

30 lnv. TG389.

31 Auguste Fraissinet, "Photographie lunaire ", La Nature, $1^{\text {er }}$ semestre 1893, pp. 201-202.

32 Auguste Fraissinet, « Photographies lunaires récemment obtenues à l'Observatoire de Paris $॥$, La Nature, $1^{\text {er }}$ semestre 1895, pp. 295-298.

33 Inv. TG391.

34 Auguste Fraissinet, « Photographies lunaires récemment obtenues à l'Observatoire de Paris », art. cit.
Précisons que les travaux de Lœwy et Puiseux relatifs à la réalisation de l'Atlas de la lune sont encore signalés en $1899^{35}$; les collections du Conservatoire s'enrichissent de nouvelles épreuves de la Lune exécutées par Lœwy en $1904^{36}$.

Prosper et Paul Henry s'attelèrent également à la photographie de Saturne ${ }^{37}$; un article, paru dans La Nature en 1884, nous renvoie aux clichés inv. 13057-2, 3 et 4, très certainement réalisés en $1885^{38}$ et enregistrés dans les collections du Conservatoire en 189839. En 1894, Auguste Fraissinet rappelle ces travaux dans un article sur la photographie des « petites planètes ${ }^{40}$ ").

Le projet de cartographier le ciel fut initié en 1884 avec les travaux de Paul et Prosper Henry. Encouragé par le premier Congrès astrophotographique de 1887 , on en annonça l'achèvement (pour ce qui concernait les étoiles jusqu'à la quatorzième grandeur) en $1912^{41}$. Charles Fabre, auteur du Traité encyclopédique de photographie, écrivait :

"La détermination exacte de la position des astres ou l'établissement d'un catalogue d'étoiles constitue certainement la branche la plus ingrate des observations astronomiques, ef cependant l'une des plus utiles, car c'est le seul moyen d'obtenir ce que l'on pourrait appeler « la Géographie du ciel ». La photographie, en fixant sur la plaque sensible la position, à une époque déterminée, de tous les astres que nous pouvons apercevoir, permet d'effectuer rapidement ce dur labeur qui n'aurait pu être fait que par plusieurs générations d'astronomes; de plus, la photographie permet d'éviter les erreurs et les omissions forcément inséparables de tout travail de cette nature ${ }^{42}$.

35 Louis Rabourdin, "La Lune et sa photographie », La Nature, $2^{\text {e }}$ semestre 1899, p. 405

36 Inv. 13635

37 Paul et Prosper Henry, "Sur l'aspect de la planète Saturne », La Nature, $1^{\text {er }}$ semestre 1884, p. 369. Les frères Henry publieront un autre article en 1886 : « La Photographie astronomique à l'Observatoire de Paris ॥, La Nature, $1^{\text {er }}$ semestre 1886, p. 23-26. 38 Les épreuves inv. 13057-2 et 3 comportent une inscription manuscrite mentionnant la date du 21 décembre 1885. L'épreuve inv. 13057-4 n'est pas datée.

39 Les épreuves inv. 13057, TG388, TG389 et TG395 entrent dans les collections du Conservatoire par donation de Prosper et Paul Henry en 1898.

40 Auguste Fraissinet, "La Photographie des petites planètes " La Nature, $2^{\mathrm{e}}$ semestre 1894, pp. 202-204.

41 Georges Brunel, La Pratique et l'art de la photographie, 1912, p. 11.

42 Charles Fabre, Traité encyclopédique de photographie 


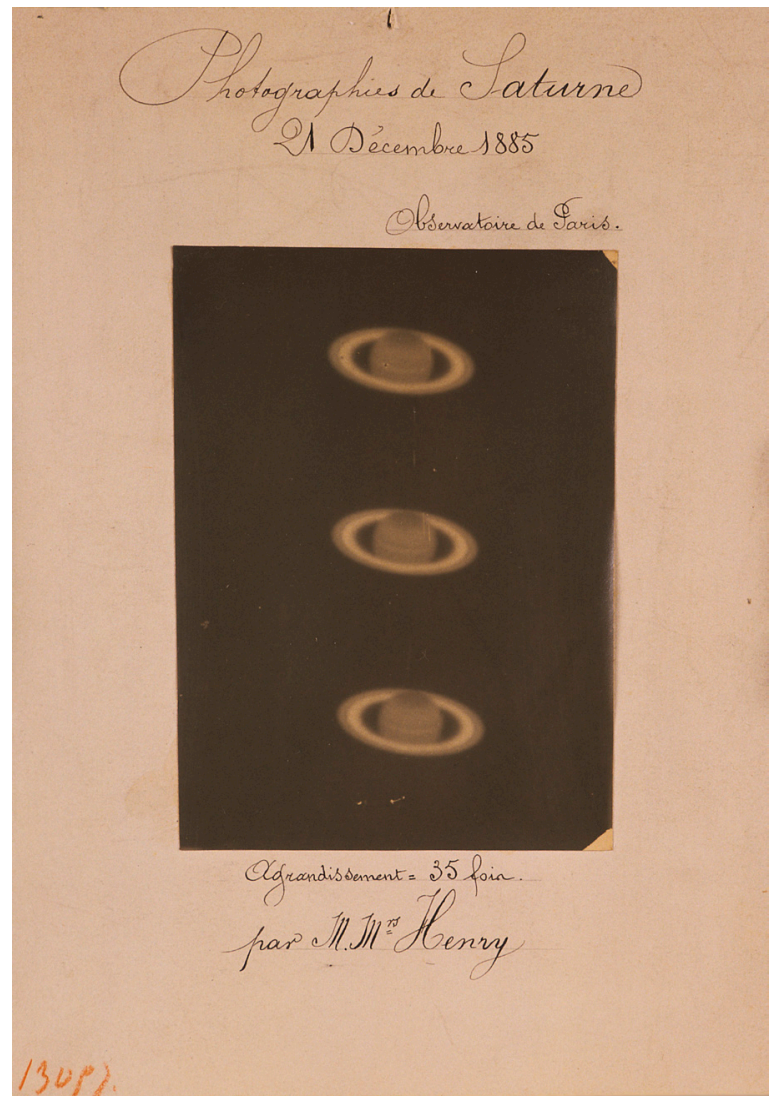

La planète Saturne, vues prises de l'Observatoire de Paris le 21 décembre 1885, Prosper et Paul Henry. Épreuve positive sur papier albuminé. Inv. 13057-2. @ Musée des arts et méfiers:Cnam /L. Karteskind

Le fonds le plus cohérent de la collection de photographies astronomiques du Conservatoire revient aux frères Henry ${ }^{43}$. Dans une note publiée dans La Nature en 189944, consacrée à un article issu de Ciel et Terre portant sur les recherches d'Edward Barnard (1857-1923), astronome à l'Observatoire de Yerkes, l'auteur rappelle l'importance de leurs travaux:

"Les frères Henry se sont activement appliqués à la photographie céleste et avec le plus brillant succès : ils ont ouvert la voie au monde astronomique dans ce domaine. Ils avaient déjà atteint le plus haut degré de perfection que les astronomes ne faisaient encore qu'entrevoir l'importance du sujet. ॥

En 1887, Gaston Tissandier écrivait déjà :

" MM. Henry ont poursuivi leurs beaux travaux de la photographie du ciel avec une grande

tome IV, 1890, pp. 195-196.

43 Voir inv. 10579, 13057, TG388, TG389, TG395.

44 La Nature, $2^{\mathrm{e}}$ semestre 1899, p. 71. persévérance et un rare succès. lls ont obtenu de remarquables résultats pour les satellites de Jupiter, l'anneau de Saturne, et les photographies de la lune. [...] Nous mentionnerons tout spécialement aujourd'hui les photographies d'étoiles dont MM. Henry ont doté l'astronomie. [...] On aura enfin une idée de ce qu'est l'infinité des mondes, quand on saura que pour représenter tout le ciel visible à la surface du globe, il ne faudrait pas moins de dix mille photographies semblables à celle que nous publions aujourd'hui, et que tout le ciel visible sur notre terre est un point dans l'immensité ${ }^{45} . \mid$.

En 1886, Ernest Mouchez consacre un article aux travaux des frères Henry ${ }^{46}$. Les vues d'étoiles inv. 10579, exécutées par Prosper et Paul Henry à l'Observatoire de Paris et signalées par l'auteur ${ }^{47}$, sont enregistrées dans les collections du Conservatoire dès 1885 . $\|$ est encore fait mention de ces vues du groupe des Pléiades en $1888^{48}$

\section{La météorologie}

Jules Janssen ${ }^{49}$ insistait en 1888 sur l'intérêt qu'il y avait à photographier les nuages et les divers phénomènes météorologiques. La photographie apportait " des éléments de discussion précieux et variés » à la météorologie, " en donnant des phénomènes des images d'ensemble [...], en permettant des mesures de distances, de hauteur, de dimensions des nuages [...], en ouvrant aux études toute une voie de mesures photométriques de la lumière des astres dans ses rapports avec l'atmosphère [...], en permettant de léguer à l'avenir un ensemble de documents utilisables ». Cependant, l'obtention de ce type d'images n'était pas sans poser des difficultés. La sensibilité des plaques photographiques dites ordinaires était sensiblement identique pour les radiations bleves et la lumière blanche.

L'interprétation des communications présentées à la Société française de photographie relatives à cette application fait apparaître une application mineure.

45 Gaston Tissandier, «LaPhotographie céleste àl'Observatoire de Paris ॥, La Nature, $1^{\text {er }}$ semestre 1887, pp. 200-202.

46 Ernest Mouchez, « Photographies astronomiques de MM. Paul et Prosper Henry II, La Nature, 1er semestre 1886, pp. 186-187. 47 Ibidem.

48 Ernest Mouchez, " Nouvelle carte photographique du groupe des Pléiades ॥, La Nature, 1er semestre 1888, pp. 355-356. 49 Jules Janssen, Bulletin de la Société française de photographie, séance du 2 mars 1888, pp. 72-75 ; « Sur l'application de la photographie à la météorologie ॥, Comptes rendus des séances de l'Académie des sciences, tome CV, p. 1164

138 Documents pour l'histoire des techniques n 18 - décembre 2009 


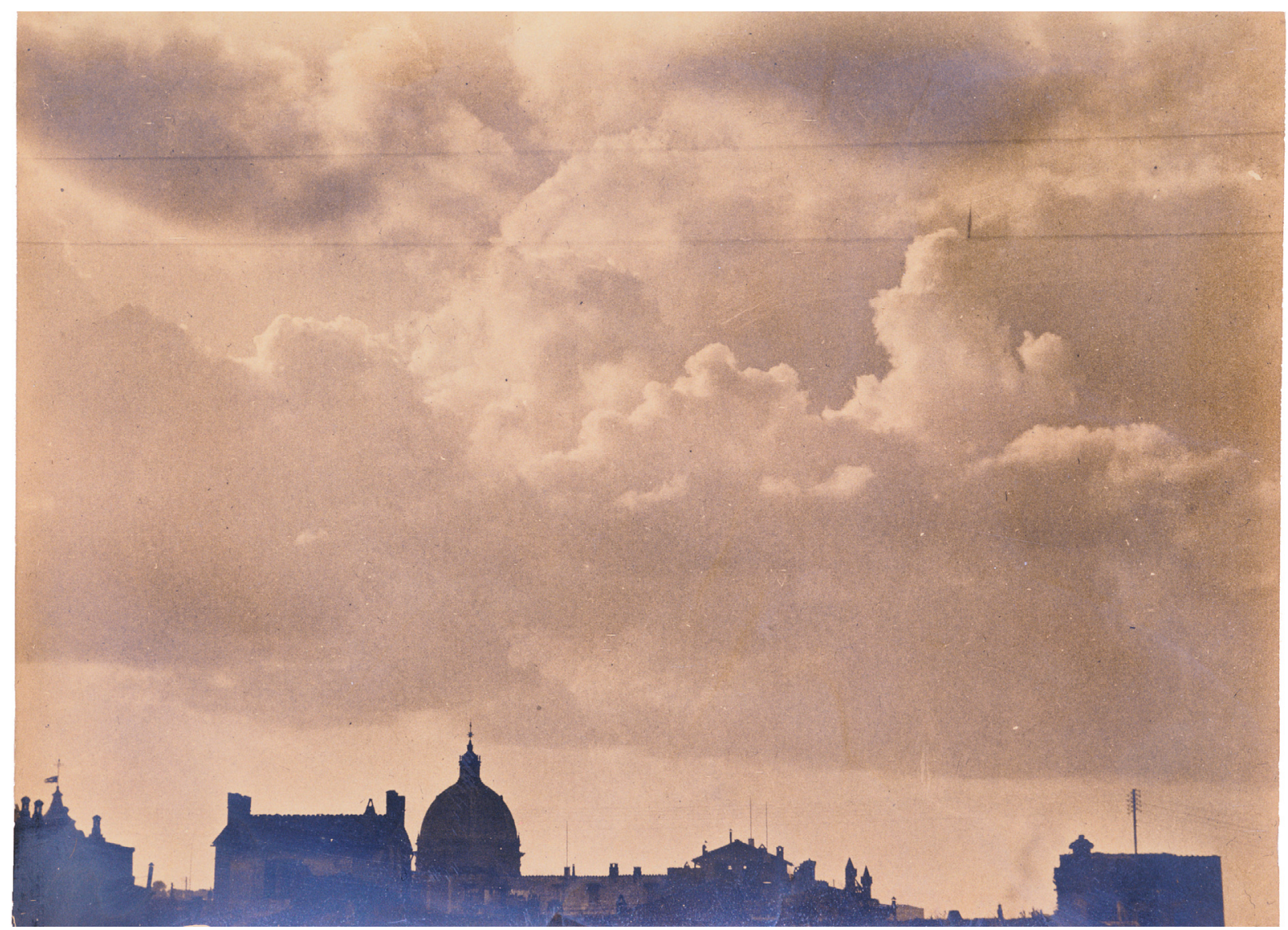

Vue issue de la série des spécimens de nuages prise à l'Observatoire du Vatican, Federico Mannucci, vers 1890. Épreuve positive sur

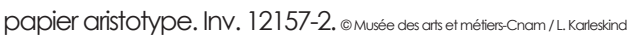

Cette dernière est toutefois liée à l'apparition et au développement des appareils photographiques enregistreurs et aux recherches en matière d'orthochromatisme.

On peut distinguer deux thèmes récurrents au sein des collections : la photographie des éclairs et celles des nuages. Mais il nous faut préalablement signaler l'allusion au magnétomètre enregistreur photographique de Brooke, entré dans les collections à la suite de l'Exposition universelle de $1851^{50}$, dans un article généraliste consacré à l'application de la photographie à la météorologie ${ }^{51}$.

\section{Les éclairs}

La Nature publie cina articles entre 1884 et 1895 sur la reproduction des éclairs par la photographie instantanée ${ }^{52}$. II y est question en 1885 des clichés

\section{Inv. 5273 à 5277.}

51 "Application de la photographie à la météorologie ", La Nature, ler semestre 1890, pp. 114-115.

52 Gaston Tissandier, « Les éclairs reproduits par la photographie instantanée 11, La Nature, 1 1er semestre 1884, pp. 76-77. Charles

instantanés ${ }^{53}$ réalisés par Charles Desquesnes, et en 1895 des clichés ${ }^{54}$ exécutés par Étienne Léopold Trouvelot (1827-1895) 55 - qui, selon Ferdinand Quénisset (1872-1951), « [avait] le premier attiré l'attention sur ce sujet $1^{56}$. Desquesnes obtint des clichés d'éclairs pendant l'orage du 13 juillet 1884. Ils furent présentés par Éleuthère Élie Mascart (1837-1908) à la Société française de physique à l'occasion de sa séance du 21 novembre 1884 où ils furent très remarqués. Cette même année, Aimé Laussedat fit part de son souhait à Desquesnes d'en obtenir un exemplaire pour les galeries du Conservatoire, proposant en retour de prendre en charge les frais de réalisation d'un agrandissement

Desquesnes, «Photographie des éclairs », La Nature, $1^{\text {er }}$ semestre 1885, p. 32. Gaston Tissandier, « Les Éclairs, leur reproduction par la photographie ॥, La Nature, 1 ${ }^{\text {er }}$ semestre 1889, p. 17-18; «Les Éclairs reproduits par la photographie ॥, La Nature, 2e semestre 1891, p. 368. Charles Édouard Guillaume, "La Durée des éclairs ॥, La Nature, $1^{\text {er }}$ semestre 1895, pp. 213-214.

53 Charles Desquesnes, "Photographie des éclairs ", La Nature, $1^{\text {er }}$ semestre 1885, p. 32. Inv. 10419 et 10776.

54 Charles Édouard Guillaume, art. cit.

55 Inv. 35732-25 à 28.

56 Ferdinand Quénisset, Applications de la photographie à la physique et à la météorologie, pp. 67-68. 
destiné à la Société française de physique. Eugène Trutat ${ }^{57}$ projeta les clichés réalisés par Desquesnes le 13 juillet 1884 à l'occasion de la conférence sur l'enregistrement des phénomènes naturels par la photographie qu'il donna au Conservatoire le 21 février 1892.

\section{Les nuages}

La photographie des nuages est tout particulièrement représentée dans la collection du Conservatoire par la série inv. 12157-2 obtenue par l'ingénieur Federico Mannucci (1848-1935) à l'Observatoire du Vatican, en 1890 ou 1891, et entrée dans les collections dès 1891. Eugène Trutat cite cette série dans sa conférence de février 1892 :

"A A'Observatoire du Vatican, M. F. Mannucci a déjà réuni une collection importante de photographies de nuages, et vous trouverez dans les collections du Conservatoire une série remarquable d'épreuves de |'habile astronome romain ${ }^{58} .11$

On apprend dans un article d'Auguste Fraissinet, publié en 1895, que le nouvel établissement, dont la direction était confiée au père Denza, allait s'occuper de photographie céleste :

"Le service scientifique de l'Observatoire du Vatican, tel qu'il a été établi par le règlement du 1er juillet 1890, se compose des branches d'études suivantes : $1^{\circ}$ astronomie, observations méridiennes et équatoriales, observations physiques diverses ; $2^{\circ}$ photographie astronomique ; $3^{\circ}$ magnétisme : $4^{\circ}$ tremblements de terre $; 5^{\circ}$ météorologie ${ }^{59} .11$

Fraissinet ajoutait que "les observations météorologiques régulières [avaient] pu commencer

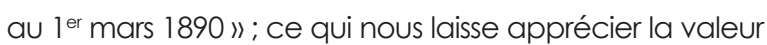
historique de ces images, offertes au Conservatoire en 1891.

La médecine et l'histoire naturelle

L'association de la photographie à la médecine et à I'histoire naturelle s'établit selon deux axes essentiels. La période de 1880 à 1920 fut la plus favorable au développement de la photographie médicale.

57 Eugène Trutat, « De l'enregistrement par la photographie des phénomènes naturels », Annales du Conservatoire national des arts et métiers, 1892, 2e série, tome IV, pp. 421-450.

58 Eugène Trutat, ibidem, p. 431. Trutat mentionne d'autre part le cliché de tornado réalisé dans le Dakota le 28 août 1884, inv. 10517, acquis par achat du Conservatoire en 1885 (p. 432). 59 Auguste Fraissinet, « L'Observatoire du Vatican », La Nature, $2^{\mathrm{e}}$ semestre 1895, pp. 19-22.
C'est à cette époque que s'imposa la photographie instantanée, que les procédés à la lumière artificielle se développèrent, que les surfaces orthochromatiques et panchromatiques furent mises au point, enfin que les rayons $X^{60}$ furent découverts.

\section{La physiologie}

L'utilisation des techniques chronophotographiques à l'étude du mouvement constitue la majorité des articles parus dans La Nature se rapportant à l'application de la photographie à la médecine. Le Conservatoire acquiert dès 1882 des épreuves faisant état des travaux du physiologiste Étienne-Jules Marey (1830-1904). Le registre de la main courante mentionne une « série de trois photographies instantanées pour l'étude de la locomotion chez les oiseaux ${ }^{61}$ » pour l'année 1882 ; une « série de trois photographies instantanées pour l'étude de la locomotion chez les oiseaux et chez l'homme ${ }^{62}$ ") en 1883 ; une " collection de trentesept épreuves photographiques se rapportant aux études de Marey sur la locomotion de l'homme et des animaux ${ }^{63}$ " en 1887 ; une " collection de onze épreuves photographiques se rapportant aux études de Marey sur la locomotion de l'homme et des animaux ${ }^{64}$ "I en 1888. La direction du Conservatoire ${ }^{65}$ accuse réception en 1888 de la livraison de onze épreuves, par Georges Demenÿ, en complément de l'envoi d'octobre 1887 d'une série extraite des Comptes rendus de l'Académie des sciences liées aux études sur la locomotion de l'homme et des animaux. Elle fait part de l'installation de l'appareil chronophotographique, construit sous la direction de Marey, dans la galerie des arts graphiques. L'appareil avait été livré en décembre 1887. Rappelons que l'établissement avait préalablement enrichi les collections d'un odographe ${ }^{66}$, donné par Marey, et d'un « explorateur de la respiration ${ }^{67}$ " acquis par achat auprès de son constructeur, la maison Breguet, au cours de l'année 1880. Les travaux de Marey font l'objet de près

60 E. Hospitalier, "Les ombres radiographiques de M. le Professeur Wilhelm Conrard Röntgen ", La Nature, $1^{\text {er }}$ semestre 1896, p. 156 : « Les premières expériences faites en France l'ont été au laboratoire de M. Le Roux, à l'Ecole supérieure de pharmacie, par M. G. Séguy, et répétées ultérieurement par MM. Les Drs Oudin et Barthélemy, Lannelongue, etc. $॥$

61 Inv. 9820.

62 Inv. 10016

63 Inv. 11056

64 Inv. 11092.

65 Une copie de la lettre adressée par la direction du Conservatoire national des arts et métiers à Étienne-Jules Marey le 4 janvier 1888 est conservée dans le dossier d'œuvre inv. 9820. 66 Inv. 9439.

67 Inv. 9475. 
d'une trentaine d'articles, publiés dans La Nature entre 1874 et 1902 ; Marey en est parfois lui-même l'auteur ${ }^{68}$. On remarquera une stricte corrélation du point de vue de la chronologie des acquisitions réalisées par le Conservatoire entre les thématiques abordées dans ces articles (vol des oiseaux, locomotion de l'homme et des animaux) et les fonds décrits dans le registre de la main courante ${ }^{69}$.

Les maladies nerveuses

Jean-Martin Charcot (1825-1893) $)^{70}$ fut, en 1878, à l'origine de la création du laboratoire de photographie médicale de la Salpêtrière. La photographie entrait officiellement dans les services hospitaliers. Ce service fut dirigé par Albert Londe (1858-1917) à partir de 1882. Londe utilisa les méthodes chronophotographiques développées par Marey. En 1883, il présentait « un appareil photoélectrique destiné à des recherches médicales ${ }^{71}$ ". Les collections du Conservatoire ne comportent pourtant aucun cliché exécuté par Albert Londe. Les appareils chronophotographiques qu'il a élaborés dans le cadre de ses recherches ont été signalés dans La Nature ${ }^{72}$. Cependant, il faut attendre la création des

68 Étienne-Jules Marey, "Le Fusil photographique ", La Nature, 1er semestre 1882, p. 326-330; " La photographie du mouvement "1, La Nature, $2^{2}$ semestre 1882, pp. 115-116; "La Station physiologique de Paris », La Nature, $2^{\mathrm{e}}$ semestre 1883 , pp. 226-230 et275-279; «Le vol des oiseaux», ibidem, pp. 35-38; "Études pratiques sur la marche de l'homme, expériences faites à la Station physiologique du Parc des Princes ". La Nature, 1er semestre 1885, pp. 119-123; « Le mécanisme du vol des oiseaux éclairé par la photochronographie ", La Nature, $1^{\text {er }}$ semestre 1888, pp. 8-14 ; «Mécanique animale, des mouvements que certains animaux exécutent pour retomber sur leurs pieds, lorsqu'ils sont précipités d'un lieu élevé ॥, La Nature, 2e semestre 1894, pp. 369-370 ; " Analyse des mouvements du cheval par la chronophotographie $"$, La Nature, $2^{\mathrm{e}}$ semestre 1898, pp. 22-26.

69 À titre de complément, signalons que deux plaques positives sur verre, représentant la chute du chat et la course du chien, ont été retrouvées sans numéro d'inventaire à l'occasion de la campagne de récolement.

70 Albert Londe, "La Photographie médicale », conférence du 24 janvier 1892, Annales du Conservatoire national des arts et métiers, 2e série, tome IV, 1893, pp. 309-344.

71 Albert Londe, Bulletin de la Société française de Photographie, séance du 4 mai 1883, pp. 123 et 125-130: "La photographie en médecine, appareil photo-électrique », La Nature, 2e semestre 1883, pp. 215-218. Inv. 11943, 16955 , 16950-1, 16958, 16959, 16960, 16961, 18069.

72 Albert Londe, " La photographie en médecine, appareil photo-électrique ", art. cit. ; "La Chronophotographie ", La Nature, $1^{\text {er }}$ semestre 1890, pp. 97-99; " La Chronophotographie », ibidem, pp. 151-154 ; « La Photochronographie dans les salles consacrées à la photographie et au cinéma en 1926 pour que le Conservatoire en fasse l'acquisition ${ }^{73}$. Signalons néanmoins le don en 1890 par son constructeur, Charles Dessoudeix, de la maquette du laboratoire photographique de l'hôpital de la Salpêtrière présentée à l'Exposition universelle l'année précédente ${ }^{74}$.

\section{L'anatomie}

Les expériences de Wilhelm Conrad Roentgen (1845-1923) ont conduit à l'une des découvertes scientifiques les plus importantes de la fin du XIXe siècle, les rayons X. Dans un article paru en 1896 dans La Nature, E. Hospitalier fait état de la portée de la découverte:

«Les récentes expériences de $M$. le professeur W. C. Röntgen, de l'Université de Würtzbourg, ont mis en émoi, depuis le commencement de l'année, le monde scientifique et aussi le gros public, généralement assez indifférent aux questions de science abstraite et de théorie pure. La forme originale et saisissante sous laquelle les expériences ont été présentées n'a pas peu contribué à ce succès, ef l'imagination aidant, la presse quotidienne a vu dans les travaux, d'ailleurs des plus intéressants, du savant allemand, une découverte à laquelle on a attaché déjà les noms les plus divers : la photographie de l'invisible; la photographie à travers les corps opaques; la photographie par les rayons cathodiques, l'électrophotographie, etc..$^{75}$ )

Le Conservatoire va acquérir des collections d'épreuves radiographiques entre 1896 et $1898^{76}$. Les premières épreuves acquises sont réalisées par Albert Peignot, préparateur au cours de physique du Conservatoire $^{77}$. Albert Londe fait référence à ses travaux dans la conférence consacrée à la radiographie qu'il donne le 15 janvier 1899 au Conservatoire :

" A titre d'exemples, voici une main injectée au moyen du mercure, puis le squelette d'un poisson conservé dans l'alcool ; c'est une pièce de collection, et avant la Radiographie il eût été nécessaire de la sacrifier pour en faire l'étude. Ce beau cliché est dû à $M$. Peignot, qui a été un des premiers

sciences médicales, le nouveau laboratoire de la Sâlpétrière ॥, La Nature, $2^{\mathrm{e}}$ semestre 1893, pp. 370-374.

73 Inv. 16957-1, 16958, 16959, 16960, 16961-2, 18069

74 Inv. 11943

75 E. Hospitalier, «Les ombres radiographiques... », La Nature $1^{\text {er }}$ semestre 1896, p. 155

76 Inv. 121913, 13088, 13059, 13089.

77 Inv. 12913 


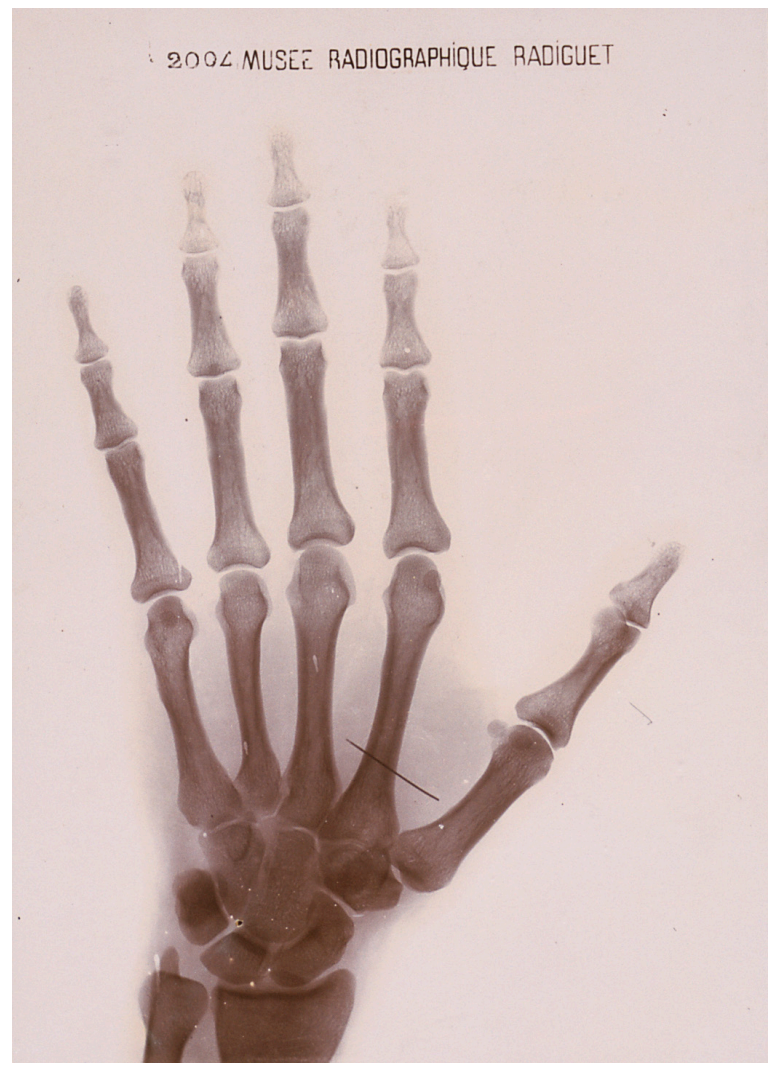

Radiographie d'une main, vue extraite du Musée radiographique, Arthur Honoré Radiguet, vers 1896. Épreuve positive sur papier aristotype. Inv. 13059-29.

certainement à obtenir des épreuves aussi fines et aussi complètes ${ }^{78}$. ॥

Les articles publiés dans La Nature sont nombreux et leur parution est récurrente, mais les expérimentateurs n'ont pas de lien direct avec les auteurs des clichés que I'on peut relever dans la collection du Conservatoire, si ce n'est Arthur Honoré Radiguet, auteur d'une série sur l'étude des matériaux.

\section{L'histoire naturelle}

La série inv. 10850, exécutée par Lachenal en 1885, fait état des recherches sur le développement de la betterave à sucre menées par Aimé Girard (1830-1898) ${ }^{79}$, professeur de chimie industrielle au Conservatoire de 1871 à 1897. Selon Émile Fleurent, «son attention avait été attirée dans cette voie par la crise qui sévissait alors sur l'industrie sucrière " .

78 Albert Londe, « La Radiographie et ses diverses applications 》, conférence du 15 janvier 1899, Annales du Conservatoire national des arts et métiers, 1899, 3e série, tome l, pp. 184-185.

79 Pierre Vigreux, notice consacrée à Aimé Girard in Claudine Fontenon et André Grelon (dir.), Les Professeurs du Conservatoire national des arts et métiers. Dictionnaire biographique, 17941955, tome I, pp. 555-566.
Ci-contre : étincelles directes obtenues par la bobine de Ruhmkorff ou la machine de Wimshurst dites «Figures de Trouvelot », Étienne Léopold Trouvelot, vers 1888. Épreuve positive sur papier aristotype. Inv. 35732-11.

D'après Pierre Vigreux :

« [...] de 1881 à 1887 Aimé Girard étudie dans une ferme dépendant de l'Institut agronomique et située à Joinville-le-Pont (Val-de-Marne), les différentes étapes du développement de la betterave, dans toutes ses parties feuilles, racines et radicelles. Ceci lui permet de définir la saccharogénie et de montrer que le sucre se forme dans les feuilles pendant la journée et migre vers la racine la nuit. II s'est attaché notamment à déterminer les dimensions du système radiculaire ${ }^{80} .11$

Signalons que Girard, répondant à la demande de Laussedat de signaler « les objets les plus indispensables pour compléter les collections correspondantes aux différents cours ${ }^{81} 1$, insiste sur l'industrie de la betterave :

"La sucrerie et la distillerie modernes sont, on peut le dire, inconnues aux visiteurs de nos galeries et, pour tout dire d'un mot, je fais au Conservatoire douze leçons sur la sucrerie sans avoir un seul modèle à mettre sous les yeux du public. ॥

II est fait état des résultats des travaux de Girard en 1886 dans La Nature ${ }^{22}$. Cette série de photographies a été offerte au Conservatoire en 1886 par Lachenal, sans doute à l'initiative de Girard. Laussedat signalait en 1899 que l'on pouvait voir dans la galerie de Chimie industrielle « des spécimens des photographies dont il se servait pour étudier le développement progressif de la plante et la formation du sucre dans les tissus ${ }^{83}$. "

80 Émile Fleurent, "La vie et les travaux d'Aimé Girard », leçon d'ouverture du cours de chimie industrielle professée au Conservatoire national des arts et métiers le 17 février 1899, Annales du Conservatoire national des arts et métiers, 1899, $3^{\text {e }}$ série, tome l, p. 135.

81 [Aimé Laussedat], Rapport sur la situation actuelle du Conservatoire national des Arts et Métiers et sur la nécessité de l'améliorer, par le Directeur de l'Etablissement, juin 1882, pp. 39-40.

82 "Composition de la betterave ", La Nature, $2^{\mathrm{e}}$ semestre 1886, p. 63.

83 Aimé Laussedat, « Inauguration du buste d'Aimé Girard au Conservatoire des arts et métiers à Paris le mercredi 20 décembre 1899 ॥, Annales du Conservatoire national des

$142 \checkmark$ Documents pour l'histoire des techniques $\mathrm{n}^{\circ} 18$ - décembre 2009 


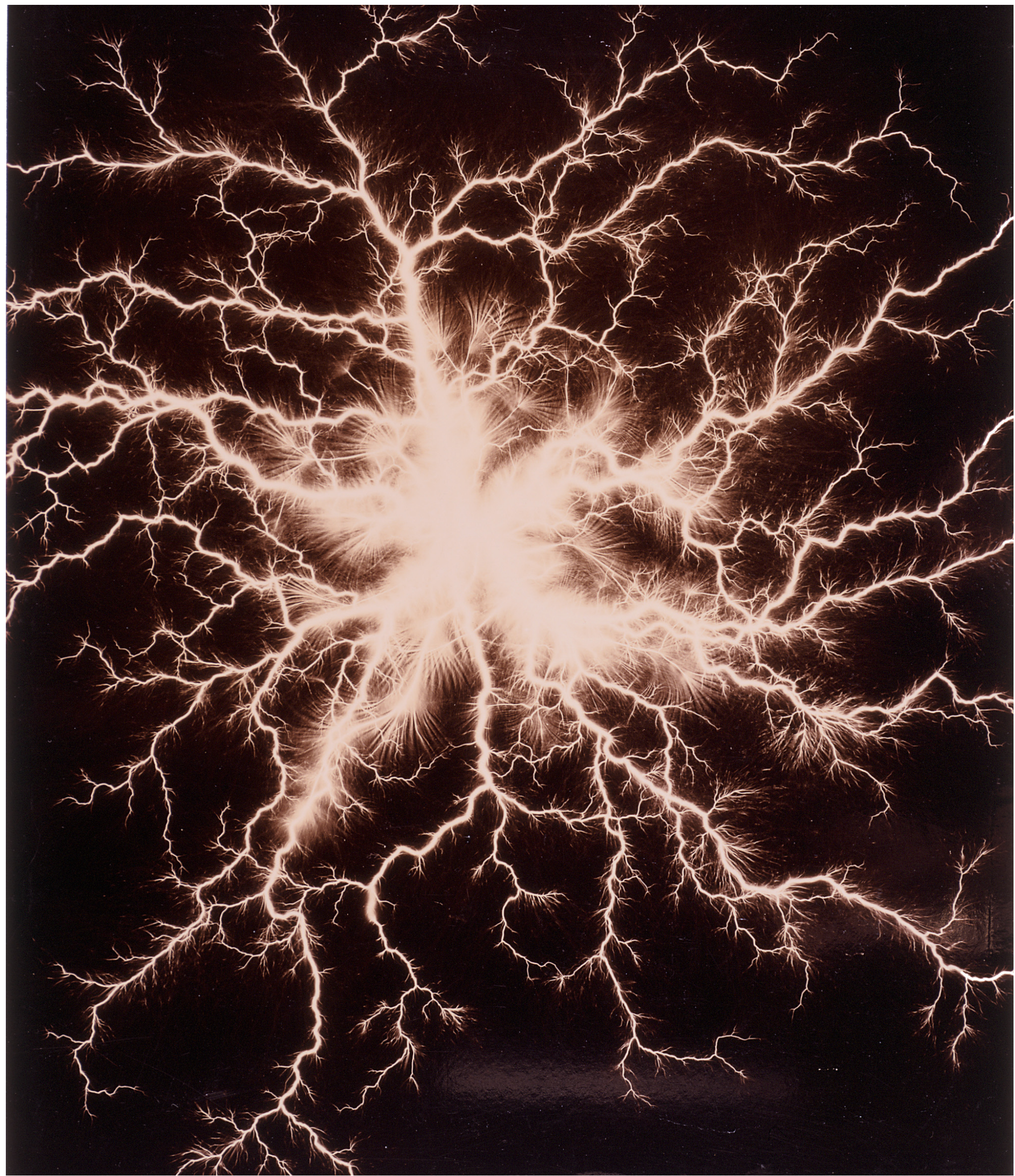


La physique

L'application de la photographie aux sciences physiques concerne l'étude des phénomènes physiques, mécaniques, calorifiques, électriques, magnétiques ou optiques. Charles Fabre justifiait cette application de la photographie dans son ouvrage de référence, le Traité encyclopédique de photographie :

"Plusieurs des phénomènes que l'on étudie en physique se manifestent sous forme du déplacement d'un ou plusieurs points matériels. La photographie, en permettant d'enregistrer la position successive de ces divers points, permet toujours de constater ce déplacement et quelquefois d'en déduire des lois ${ }^{84} . " 1$

L'étude des phénomènes mécaniques inclut ainsi la photographie des projectiles pendant le tir ou bien la photographie de la veine liquide. On regroupait sous le terme d'« application de la photographie aux phénomènes calorifiques » la mesure des coefficients de dilatation, la mesure des températures. On entendait par «l'étude des phénomènes électriques et magnétiques " la mise au point des électromètres enregistreurs permettant l'enregistrement des variations électriques de l'air ou de l'électricité atmosphérique, la photographie de l'étincelle électrique et des éclairs, enfin l'enregistrement des phénomènes magnétiques. L'application de la photographie à l'étude des sciences physiques consacra le rôle de la photographie en tant qu'instrument scientifique. Grâce à ses possibilités en matière d'enregistrement, la photographie va se substituer à des techniques imparfaites, comme le dessin, et constituer des documents propices à l'interprétation des phénomènes physiques. Cette application reflète tout particulièrement les progrès du système technique photographique générique en matière d'instantané.

\section{L'étincelle électrique}

La Nature ne consacre pas moins de six articles à la question des étincelles et effluves électriques, dont les parutions s'étalent de 1884 à 189785 .

arts et métiers, 1900, 3e série, tome II, p. 6.

84 C. Fabre, Traité encyclopédique de photographie, 1890, tome IV, p. 282

85 Gaston Tissandier, « Étincelles électriques reproduites par la photographie instantanée ॥, La Nature, 1er semestre 1884 p. 180 ; Gabriel Mareschal, « Reproductions photographiques sans appareil 11, La Nature, $2^{2}$ semestre 1886, pp. 139-140 ; Ch. Moussette, "Photographie des phénomènes électriques», La Nature, $2^{\mathrm{e}}$ semestre 1888, p. 192 ; « Photographie des
Le constructeur d'instruments scientifiques Eugène Adrien Ducreteł (1844-1915) réalise des expériences dans ce domaine au début des années 1880. En 1884, il donne au Conservatoire une série d'épreuves sur papier albuminé et les clichés transparents correspondants, dont les négatifs ont été obtenus sans objectif à l'aide d'une bobine d'induction ${ }^{86}$. I soulignait l'intérêt des détails contenus sur les clichés transparents, lesquels n'étaient pas visibles sur les tirages. Cette série sur verre n'est malheureusement pas parvenue jusqu'à nous (" détériorée ", elle a été "supprimée " en 1975, comme le précise la main courante). Eugène Ducretet offre à nouveau, en 1885, une série d'épreuves différentes sur papier albuminé, présentées à l'occasion de l'Exposition universelle d'Anvers ${ }^{87}$. Les expériences de Ducretet sont rapportées en 1886 dans cet article de Gabriel Mareschal :

"Lorsqu'on veut reproduire une étincelle ou un effluve électrique au moyen des appareils photographiques ordinaires, on obtient des résultats très imparfaits. II y a environ deux ans, M. Ducretet, le constructeur bien connu, a eu l'idée d'impressionner des plaques au gélatinobromure en faisant directement la décharge dans leur voisinage. A cet effet il a construit une cage en verre rouge munie à l'intérieur de supports pour les plaques, et de conducteurs de différentes formes. Il a par ce moyen obtenu des résultats très remarquables ${ }^{88} .1$

C. Moussette ${ }^{89}$ mentionne encore ces travaux précurseurs en la matière dans un article paru en 1888 Ferdinand Quénisse ${ }^{90}$ fait quant à lui référence à ces résultats dans un ouvrage consacré aux applications de la photographie à la physique et publié dans les premières années du XXe siècle :

étincelles électriques I), La Nature, $1^{\text {er }}$ semestre 1889, p. 304 ; Étienne Léopold Trouvelot, « La Photographie appliquée à l'étude de l'étincelle électrique ॥, La Nature, $2^{\mathrm{e}}$ semestre 1889, p. 109-110; G. Mareschal, « Photographie d'effluves humain et magnétique "I, La Nature, $2^{2}$ semestre 1897, p. 349.

86 Inv. 10194. Ces clichés ont été donnés l'année même de leur obtention.

87 Inv. 10702

88 G. Mareschal, « Reproductions photographiques sans appareil », art. cit.

89 C. Moussette, «Photographie des phénomènes électriques », art. cit.

90 Ferdinand Quénisset, op. cit., p. 37.

$144 \checkmark$ Documents pour l'histoire des techniques $\mathrm{n}^{\circ} 18$ - décembre 2009 


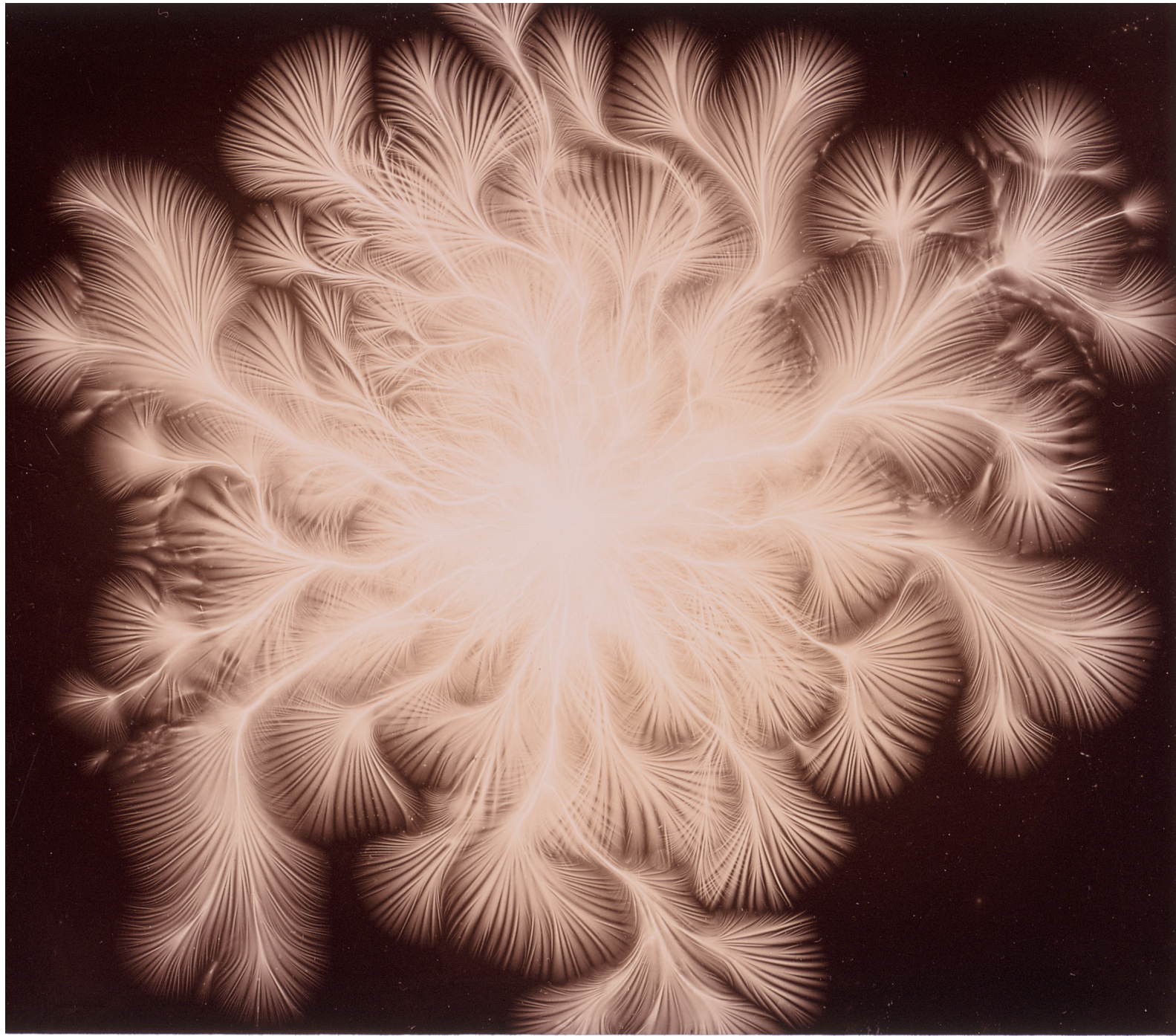

Étincelles directes obtenues par la bobine de Ruhmkorff ou la machine de Wimshurst dites " Figures de Trouvelot ", Étienne Léopold Trouvelot, vers 1888. Épreuve positive sur papier aristotype. Inv. 35732-10. @ Musée des arts et méfiers-Cnam / M. Favareille

« M. Ducretet obtint, il y a quelques années, de magnifiques épreuves sans avoir recours à un appareil de photographie. II faisait jaillir une belle étincelle dans une chambre noire, tout contre la plaque sensible. On obtient alors un trait de feu sinueux qui est absolument identique à l'éclair. "l

Nous avons retrouvé, dans le cadre de notre opération de récolement, de conservation préventive et de restauration des collections photographiques, une enveloppe portant les noms de l'astronome Étienne Léopold Trouvelot et d'Aimé Laussedat et contenant des épreuves, sans numéro d'inventaire. Ces épreuves, représentant des étincelles et effluves électriques mais également des éclairs, auraient été données pour les collections du Conservatoire par Aimé Laussedat en 1905, comme peut l'attester la date inscrite sur l'enveloppe. Un numéro d'inventaire provisoire a été attribué à cette série ${ }^{91}$, en attendant de retrouver le numéro d'origine éventuel - nous n'avons cependant identifié aucune mention correspondante sur la main courante. Nous avons toutefois identifié leur contexte de réalisation. Pour Étienne Léopold Trouvelot, il s'agissait, d'après une communication sur ses travaux à l'Académie des sciences, de «répéter les expériences si intéressantes faites en 1884 par M. E. Ducretet ${ }^{92}$ \%. Dans un article consacré à la forme des décharges électriques sur les plaques photographiques, publié dans La Lumière électrique ${ }^{93}$ en 1888, Trouvelot remerciait d'ailleurs Eugène Ducretet pour sa collaboration :

\section{Inv. 35732}

92 Étienne Léopold Trouvelot, « La Photographie appliquée à l'étude des décharges électriques ", Comptes rendus des séances de l'Académie des sciences, 7 décembre 1888, p. 684. 93 Étienne Léopold Trouvelot, « Sur la forme des décharges électriques sur les plaques photographiques ", La Lumière 
"L Les instruments qui m'ont servi pour obtenir les photographies qui ont servi de base à cette étude, ont été gracieusement mis à ma disposition par M. Ducretet, auquel je suis heureux d'exprimer publiquement mes sincères remercîments [sic] à M. Ernest Roger, ingénieur de la maison Ducretet qui m'a aidé dans toutes mes expériences avec un zèle et une intelligence dignes de tous éloges. ॥

Cet autre extrait, provenant d'un article de Trouvelot ${ }^{94}$ paru en 1889 dans La Nature, expose le protocole de réalisation de ces épreuves:

"Les images données par la grande machine de Holtz, du Conservatoire des arts et métiers, mise à notre disposition par le bienveillant directeur de cette institution, M. le colonel Laussedat, ainsi que celles que j'ai obtenues avec la grande machine à douze plateaux de Wimshurst, construite par M. Ducretet et maintenant en exhibition au Palais des arts libéraux, diffèrent entre elles, comme elles diffèrent également des images fournies par d'autres appareils. ॥

Rappelons que les collections du Conservatoire comportent deux exemplaires de la machine électrique de Wilhelm Holtz, construites respectivement par Heinrich Ruhmkorff et Eugène Ducretet. La première a été acquise par achat en 1867 à la suite de l'Exposition universelle de Paris ${ }^{95}$, la seconde en 1882, probablement à la suite de l'Exposition internationale d'électricité de $1881^{96}$. Deux machines électriques de James Wimshurst97, dont l'une au moins a été construite par Ducretet ${ }^{98}$, figurent quant à elles dans les collections. Elles ont été acquises par achat en 1889, très certainement à la suite de leur présentation au public dans le cadre de l'Exposition universelle. Trouvelot analysait les images :

"À première vue on reconnaît sur nos clichés un fait important et capital qui domine tous les autres. Les images engendrées par la décharge de l'électricité positive sont dissemblables, et ne ressemblent en aucune manière aux images engendrées par la décharge de l'électricité négative. Les premières,

électrique, tome XXX, octobre-novembre 1888, pp. 269-273. 94 Étienne Léopold Trouvelot, « La Photographie appliquée à l'étude de l'étincelle électrique ", La Nature, $2^{2}$ semestre 1889, pp. 109-110.

95 Inv. 7680.

$96 \operatorname{lnv} .9756$.

97 Inv. 11625-1 et 2.

98 Inv. 11625-1. sinueuses et singulièrement ramifiées, ressemblent à certains lichens, à certaines algues; tandis que les secondes, à ligne souvent brisée, rappellent par leur forme les feuilles élégantes de certains palmiers.

"Bien que d'une manière générale on puisse affirmer que toutes les étincelles électriques de même nom, qu'elle [sic] qu'en soit la source, se ressemblent, et ont un caractère, un air de famille tout à fait indiscutable, elles portent cependant, jusqu'à un certain point, un cachet individuel plus ou moins marqué qui fait qu'une étincelle, donnée par une certaine machine de différente grandeur ou de différente construction. La différence de forme tient peut-être à une question de quantité de tension ${ }^{99} .11$

Il illustrait son propos en montrant " deux cas extrêmes de variations de l'étincelle avec l'emploi d'une bobine de Ruhmkorff et d'une machine de Wimshurst ", dont on retrouve les équivalents dans la série inv. 35732. Ferdinand Quénisset fait allusion à ces expériences :

« Enfin l'éminent astronome Trouvelot, qui s'est beaucoup occupé aussi des phénomènes électriques, a obtenu de superbes photographies des effluves qui se dégagent autour des pôles d'une bobine de Ruhmkorff ou d'une machine statique. Ces curieuses épreuves, datant de 1888, ont été très remarquées et sont même connues dans la science sous le nom de figures de Trouvelot ${ }^{100}$. $)$

\section{La balistique}

Dans le domaine de la balistique, les travaux d'Ernest Mach (1838-1910) et de Salcher sont rapportés dans trois articles, parus successivement en 1888, 1890 et $1896^{101}$. Les images de Mach sont acquises par le Conservatoire entre 1890 et 1893. II est notamment fait mention, dans l'article de $1888^{102}$, des lithographies dessinées à la main par Reisek d'après les épreuves photographiques obtenues par Mach et Salcher ${ }^{103}$, qui nous renvoient à la planche inv. 11988, entrée en 1890 dans les collections. En ce qui concerne les épreuves

99 Étienne Léopold Trouvelot, « La Photographie appliquée à l'étude de l'étincelle électrique ॥, art. cit., p. 110.

100 Ferdinand Quénisset, op. cit., p. 37.

101 Gaston Tissandier, «Photographies des projectiles pendant le tir II, La Nature, $1^{\text {er }}$ semestre 1888, pp. 210-211 et 387-388; La Nature, $1^{\text {er }}$ semestre 1890, p. 264 ; " Photographie d'une balle en mouvement "), La Nature, $2^{\mathrm{e}}$ semestre 1896, p. 320. 102 Gaston Tissandier, «Photographies des projectiles... », art. cit. 103 Gaston Tissandier, ibidem, p. 387.

$146 \checkmark$ Documents pour l'histoire des techniques $\mathrm{n}^{\circ} 18$ - décembre 2009 
inv. 12044, une lettre de Laussedat à Gaston Tissandier, datée du 6 décembre 1890, fait état de son souhait de faire l'acquisition d'épreuves de Mach. Gaston Tissandier sert là encore d'intermédiaire. Ernest Mach répond favorablement à cette sollicitation expliquant que "la plupart des meilleurs [épreuves] est déjà dissipée par tout le monde » et qu'il fera donc copier les plus belles. On trouve une trace de ces acquisitions dans l'ouvrage de Ferdinand Quénisset104, qui opérait à l'Observatoire de Juvisy. Quénisset rapportait, en s'appuyant sur un schéma, le mode opératoire défini par Mach et Salcher en 1890 pour obtenir des photographies des projectiles ${ }^{105}$ et ajoutait :

" On peut voir les premières épreuves obtenues par MM. Mach et Salcher au Conservatoire des Arts et Métiers, à Paris, dans la salle consacrée aux applications de la Photographie ${ }^{106} .11$

Les collections du Conservatoire renferment une autre série d'épreuves et d'objets ${ }^{107}$, entrés en 1883, significatifs des travaux du lieutenantcolonel Hippolyte Sebert (1839-1930), directeur du Laboratoire central d'artillerie de la Marine. Ses expériences, réalisées en 1880 à la poudrerie de Sevran-Livry, sont détaillées dans un article de La Nature en $1881^{108}$. II s'agissait de déterminer la loi du mouvement :

"La connaissance de cette loi présente un grand intérêt pour le service de l'artillerie, car elle permettrait, dans chaque cas particulier, de déterminer la succession des pressions développées dans l'âme pendant la durée de

104 Ferdinand Quénisset, op. cit.

105 Ferdinand Quénisset, ibidem, pp. 15-16.

106 Ferdinand Quénisset, ibid., p. 16.

107 Inv. 9870

108 L. R., « ¿ Détermination de la loi du mouvement d’un projectile dans l'âme d'une pièce et dans un milieu résistant, Projectiles enregistreurs Sebert 11, La Nature, 1 ${ }^{\text {er }}$ semestre 1881, pp. 411-414. la combustion de la charge de poudre et, par suite, fournirait les éléments nécessaires, tant pour améliorer la fabrication et le mode d'emploi de la poudre que pour arriver au meilleur mode de construction des bouches à feu ${ }^{109}$. 11

Sebert utilisa le chronographe à diapason et à enregistreurs électromagnétiques mis au point avec la collaboration de Marcel Deprez (1843-1918), professeur d'électricité industrielle au Conservatoire.

\section{Le journaliste concluait :}

"La conception de M. Sebert ne représente donc pas seulement une idée originale ; les expériences sont concluantes, et I'on peut dire que son appareil constitue une acquisition de premier ordre pour les sciences qui se rattachent à l'artillerie ${ }^{110}$. "l

Le contexte de l'acquisition de cette collection est signifié dans une lettre du 4 décembre 1882 de Laussedat à Sebert. Aimé Laussedat l'informe de la réorganisation achevée de la galerie de physique et de l'installation du " grand tableau dont M. le Ministre de la Marine et des Colonies nous ont autorisé à disposer en faveur de nos collections, et qui représente le champ de tir de la poudrerie de Sevran-Livry ". Laussedat rappelle la promesse de don du ministre de la Marine de deux projectiles enregistreurs (calibres 24 et 10 centimètres) "'l' et d'une " série de photographies représentant les nouveaux appareils balistiques dont fait usage le service de l'artillerie de la marine " et demande à quel moment le Conservatoire pourra en disposer.

Projectile en mouvement, Ernst et Ludwig Mach, 1893. Épreuve positive sur nitrate de cellulose. Vue extraite de la série inv. 12427. - Musée des arts et métiers-Cnam / M.Favareille

109 Ibidem, p. 411.

110 lbid., p. 414.

111 Inv. 9870-2 et 3. 


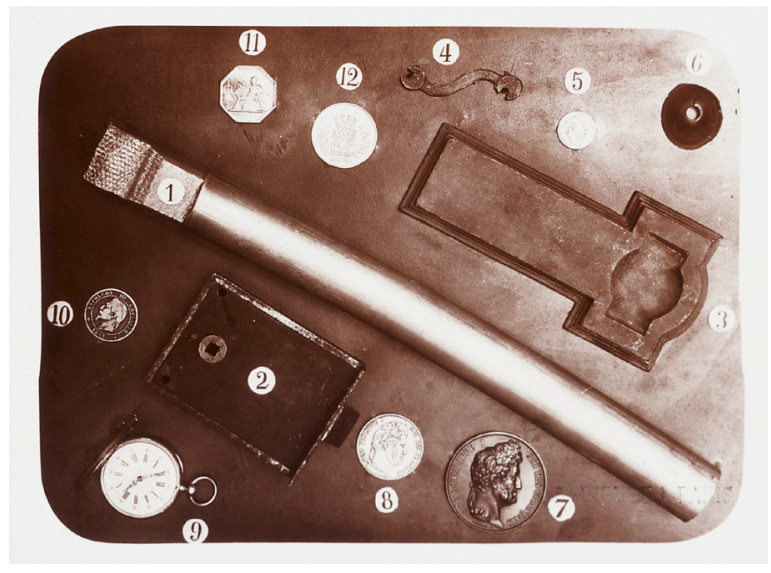

\section{L'étude des matériaux}

La série d'épreuves inv. 13059, réalisée par Arthur Honoré Radiguet, entre dans les collections en 1898. Radiguet a déposé dès 1896 plusieurs noms de marques dans le domaine de la radiographie ; ces dépôts témoignent de son implication en tant que constructeur dans ce domaine particulier de I'instrumentation scientifique. Les épreuves concernent la médecine mais surtout l'étude des matériaux. Le physicien Charles Édouard Guillaume (1861-1938) fait référence à ces expériences dans un article publié dans La Nature en 1897112. II illustre son propos à l'aide d'images que l'on retrouve dans cette série ${ }^{113}$, livre le mode opératoire et la lecture qui doit être faite des images obtenues:

«Les figures 2 et 3 [fig. 2 : Photographie montrant la disposition des objets dans la radiographie précédente, tels qu'ils sont vus du tube de Crookes - Fig. 3 : Photographie des mêmes objets, vus à travers la plaque. Disposition semblable à celle que montre le positif de la radiographie] indiquent la disposition des objets radiographiés : une tige ronde d'aluminium, une serrure, le socle en fonte d'une petite machine recouvrant une pièce de monnaie, une petite clef d'écrou, diverses médailles ou monnaies, un éclat d'ébonite et une montre à boîtier d'or.

"Ces objets ont été photographiés, vus d'abord du côté du tube, puis du côté de la plaque. Si l'on passe de la figure 3 à la radiographie obtenue à l'aide d'un tube très dur et très pénétrant, et avec une pose d'une heure, on identifiera aisément tous les objets, à l'exception du disque d'ébonite

112 Charles Édouard Guillaume, "Les rayons X et les métauX; les rayons $X$ ef la douane ", La Nature, $2^{\text {e }}$ semestre 1897 , p. 147-150.

113 Inv. 13059-15 à 17.

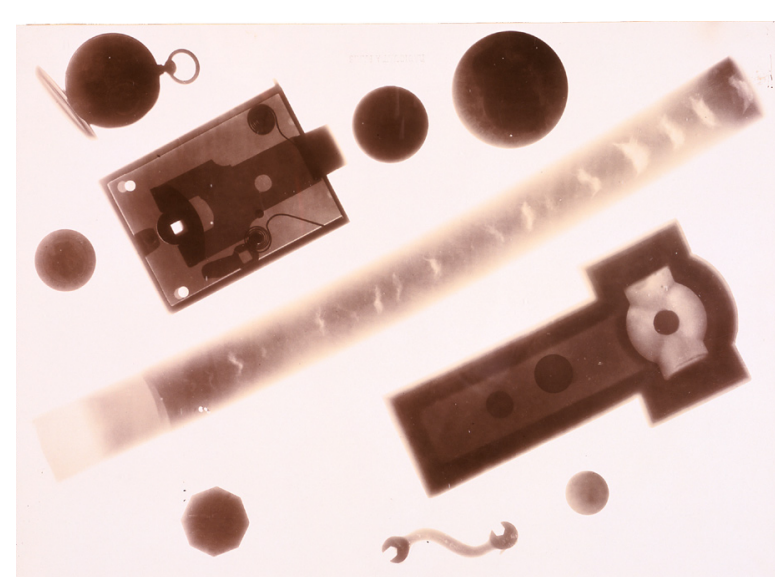

Tige d'aluminium, serrure, socle en fonte, clef d'écrou, éclat d'ébonite, boîtier de montre en or, médailles et monnaies, vues photographique et radiographique, Arthur Honoré Radiguet, vers 1896. Épreuves positives sur papier aristotype. Inv. 13059-00016 et 13059-0015. @ Musée des arts et métiers-Cnam / P. Harvey

$\mathrm{n}^{\circ} 6$ et de la médaille d'aluminium $\mathrm{n}^{\circ} 12$, dont la radiographie n'a pas gardé la moindre trace. « Les monnaies et médailles laissent apercevoir les deux faces de leur frappe, la montre laisse deviner ses rouages, tandis qu'on voit très exactement et avec vigueur tous les organes de la serrure, ainsi que les diverses épaisseurs de la garniture de métal avec la pièce de monnaie cachée dessous. La tige d'aluminium présente un intérêt tout particulier. Elle montre, en divers endroits, des parties claires se détachant bien nettement sur le fond sombre, formé par l'ensemble de la barre. La radiographie nous révèle ici des creux du métal, des défauts de continuité qu'on n'aurait découverts sans cela qu'en sciant la tige dans le sens de la longueur.

"Dans cette seule opération, M. Radiguet a accumulé des difficultés, montrant ainsi combien la nouvelle méthode est élastique, plutôt que de chercher, par des opérations séparées, la perfection dans l'examen de chacun des objets. "

On retrouve encore quelques-unes de ces radiographies ${ }^{114}$ dans le texte, publié dans les Annales du Conservatoire national des arts et métiers, de la conférence d'Albert Londe ${ }^{115}$ en 1899. Londe s'appuie en effet sur les travaux de Radiguet au cours de cette conférence, lorsqu'il évoque les progrès réalisés dans

114 Albert Londe, "La Radiographie et ses diverses applications ॥, op. cit., p. 175 : inv. 13059-1, 2, 4, 8, 34, 38 ; p. 179 : inv. 13059-10, 11 ; p. $189: 13059-15$ à 17 ; p. $190: 13059-16$ à 24. $115 \mathrm{lbidem}$.

$148 \checkmark$ Documents pour l'histoire des techniques $\mathrm{n}^{\circ} 18$ - décembre 2009 
le cadre de la radiographie appliquée à l'étude des matériaux :

« [...] on peut radiographier des pièces métalliques, constater les détails de leur structure, révéler leurs défauts non apparents. En voici quelques exemples typiques que nous devons à l'obligeance de M. Radiguet (fig. 28) : une serrure dont on aperçoit le mécanisme, une barre d'aluminium qui contient de nombreuses souffures internes, une montre dont tous les organes deviennent visibles à travers le boîtier (fig. 29) [...]. ॥

L'étude des conditions dans lesquelles s'est constitué le fonds de photographies scientifiques du musée confirme les liens que le Conservatoire des arts et métiers entretenait avec les milieux scientifiques et les sociétés savantes au XIXe siècle. La qualité des auteurs et la provenance des articles publiés dans La Nature, organe majeur de vulgarisation des connaissances à cette époque, nous ramènent à ces réseaux de sociabilité parfaitement identifiés. Il arrive que Gaston Tissandier ait un rôle d'intermédiaire entre les savants expérimentateurs et les autorités du Conservatoire. Mais en règle générale, ces dernières font jover leurs relations institutionnelles et/ou individuelles pour procéder à l'acquisition des spécimens qui alimenteront les collections.

En effet, dans ce cas précis, le rôle du Conservatoire en matière de veille technologique se manifeste lorsqu'il s'agit d'aménager une nouvelle galerie d'exposition, consacrée aux arts graphiques et à la photographie. On soulignera toutefois que certaines épreuves sont exposées dans d'autres galeries de l'institution. La constitution de collections en vue de l'exposition au public distingue le Conservatoire des institutions et des sociétés savantes, également dépositaires de collections, rassemblées à d'autres fins, et notamment de l'Académie des sciences, de la Société d'encouragement pour l'industrie nationale, ou de la Société française de photographie.

Enfin, compte tenu de la nature de la collection du Conservatoire et de l'absence de politique des musées de la photographie dans ce domaine, l'acquisition d'épreuves de photographie scientifique peut constituer aujourd'hui un axe cohérent d'enrichissement et asseoir une spécificité des collections du Musée des arts et métiers. 